\title{
Physicochemical and Antioxidant Properties of Chitosan Films Incorporated with Cinnamon Oil
}

\author{
Marco A. López-Mata, ${ }^{1,2}$ Saul Ruiz-Cruz, ${ }^{1}$ Norma Patricia Silva-Beltrán, ${ }^{1,2}$ \\ José de Jesús Ornelas-Paz, ${ }^{3}$ Víctor Manuel Ocaño-Higuera, ${ }^{4}$ Francisco Rodríguez-Félix, ${ }^{5}$ \\ Luis A. Cira-Chávez, ${ }^{1}$ C. L. Del-Toro-Sánchez, ${ }^{6}$ and Keiko Shirai ${ }^{7}$ \\ ${ }^{1}$ Departamento de Biotecnología y Ciencias Alimentarias, Instituto Tecnológico de Sonora, 5 de Febrero 818 Sur, \\ 85000 Ciudad Obregón, SON, Mexico \\ ${ }^{2}$ Departamento de Ciencias de la Salud, Universidad de Sonora, Campus Cajeme, Boulevard Bordo Nuevo, \\ Ejido Providencia, 85040 Cajeme, SON, Mexico \\ ${ }^{3}$ Centro de Investigación en Alimentación y Desarrollo A.C., Avenida Río Conchos $s / n$, Parque Industrial, \\ 31570 Cuauhtémoc, $\mathrm{CHIH}$, Mexico \\ ${ }^{4}$ Departamento de Ciencias Químico Biológicas, Universidad de Sonora, Encinas y Rosales s/n, 83000 Hermosillo, SON, Mexico \\ ${ }^{5}$ Departamento de Investigación y Posgrado en Alimentos, Universidad de Sonora, Boulevard Luis Encinas y \\ Rosales s/n Colonia Centro, 83000 Hermosillo, SON, Mexico \\ ${ }^{6}$ Departamento de Ciencias Médicas y de la Vida, Centro Universitario de la Ciénega, Universidad de Guadalajara, \\ 47810 Ocotlán, JAL, Mexico \\ ${ }^{7}$ Departamento de Biotecnología, Laboratorio de Biopolimeros, Universidad Autónoma Metropolitana, \\ Avenida San Rafael Atlixco No. 186, Colonia Vicentina, 09340 Ciudad de México, DF, Mexico
}

Correspondence should be addressed to Saul Ruiz-Cruz; sruiz@itson.edu.mx

Received 18 February 2015; Revised 13 April 2015; Accepted 15 April 2015

Academic Editor: Guiping Ma

Copyright ( 2015 Marco A. López-Mata et al. This is an open access article distributed under the Creative Commons Attribution License, which permits unrestricted use, distribution, and reproduction in any medium, provided the original work is properly cited.

\begin{abstract}
Chitosan films (CF) with cinnamon bark oil (CO) incorporated at $0 \%$ (control), $0.25 \%, 0.5 \%$, and $1.0 \% \mathrm{v} / \mathrm{v}$ were prepared by an emulsion method. The films were characterized based on their physical properties (solubility, water vapor permeability, optical property, and microstructure) and antioxidant properties (DPPH, ABTS, and its protective effects on human erythrocytes). The results showed that the incorporation of 0.5 and $1.0 \%$ of CO into the CF significantly decreased its solubility to $22 \%$ of the control $(p<0.05)$. The water vapor permeability of the CF-CO was significantly reduced to $40 \%$ with low concentrations of CO $(0.25 \%)$ incorporated into the CF. In general, the films presented a yellow coloration and an increase in transparency with the incorporation of CO into the CF. It was also observed that the incorporation of CO increased the antioxidant activity between 6.0-fold and 14.5fold compared to the control, and the protective capacity against erythrocyte hemolysis increased by as much as $80 \%$.
\end{abstract}

\section{Introduction}

Consumers now demand foods that contain low levels or are free of synthetic chemical preservatives [1]. Some alternatives have aimed to use matrices based on proteins, lipids, and carbohydrates that present film formation properties [2]. Chitosan is a carbohydrate consisting of $\beta$-(1-4)-D-glucosamine and $\beta$-(1-4)-N-acetyl-D-glucosamine, obtained from the thermo-alkaline deacetylation of chitin. This biopolymer is viscous, biodegradable, nontoxic, antigenic, antimicrobial, and biocompatible [3]. These characteristics make it suitable for the development of diverse applications in medicine, agriculture, and the food industry [4]. In the food industry, chitosan has been used as a natural preservative due to its film formation properties. It has been shown that chitosan films can create a semipermeable barrier that can reduce breathing, retard microbial growth, and offer other protective qualities in fruits and vegetables [5]. In addition, its use as 
a preservative in food products has been considered safe [6]. However, due to its hydrophilic nature, one of the main disadvantages of the CF is its limited action as a barrier against humidity. Some alternatives that have been used to improve the properties of chitosan have been the incorporation of diverse bioactive compounds such as vitamins, lipids, minerals, drugs, proteins, colorants, and essential oils [7, 8].

Cinnamon bark essential oil (CO) contains a great variety of chemical compounds (mainly cinnamaldehyde, cinnamic acid, coumaric acid, cinnamyl alcohol, and eugenol) that present antioxidant and antimicrobial activities in an individual and collective manner [9]. In the food industry, $\mathrm{CO}$ has been used as a flavoring and preservative $[10,11]$. However, its direct application to food as a preservative has been limited, as it can be lost during storage due to its high volatility and reactivity with diverse food components [12]. The hydrophobic nature of $\mathrm{CO}$ could be exploited to make CF a better barrier against humidity, and in turn the chitosan matrix could conserve the properties of $\mathrm{CO}$, which could improve the physical and antioxidant characteristics of CF. However, the incorporation of $\mathrm{CO}$ into $\mathrm{CF}$ could also generate undesired changes within the CF. Therefore, the goal of this study was to characterize the physical (solubility, water vapor solubility (WVP), and optical), antioxidant (DPPH (2,2-diphenyl-1-picrylhydrazyl), ABTS (2,2' -azinobis(3-ethylbenzothiazoline-6-sulphonic acid)), and protective effects on human erythrocytes) and microstructural properties of CF surfaces containing CO incorporated by an emulsion method.

\section{Materials and Methods}

2.1. Chitosan Preparation. Chitosan was obtained by the thermo-alkaline deacetylation of shrimp chitin according to the methodology proposed by Hongpattarakere and Riyaphan [13]. For this purpose, $1 \mathrm{~g}$ of chitin was homogenized with $15 \mathrm{~mL}$ of $\mathrm{NaOH}(50 \% \mathrm{w} / \mathrm{v})$ at $95^{\circ} \mathrm{C}$ for $2 \mathrm{~h}$. The degree of chitosan acetylation obtained was 34\%, with an average molecular weight of $128 \mathrm{kDa}$. The chitosan was characterized in the Polymers Laboratory of the Biotechnology Department at the Metropolitan Autonomous University, Mexico, DF.

2.2. FC Preparation and CO Incorporation. A chitosan solution was prepared by dissolving $0.4 \mathrm{~g}$ of chitosan in $20 \mathrm{~mL}$ of acetic acid $(1 \% \mathrm{v} / \mathrm{v})$ and homogenizing the solution for $4 \mathrm{~min}$ at 15,500 rpm (Ika Ultra-turrax T 18 basic, Germany). Subsequently, glycerol $(200 \mu \mathrm{L})$ was added as plasticizer and agitated under similar conditions. Before the incorporation of the CO (cinnamon bark oil FCC, Sigma-Aldrich), the chitosan solution was emulsified with tween $80(1 \% \mathrm{v} / \mathrm{v})$ as a surfactant. For this study, 0\% CO was used as the control, and three levels of CO incorporation were tested: $0.25 \%$, $0.5 \%$, and $1.0 \%(\mathrm{v} / \mathrm{v}) \mathrm{CO}$ in chitosan solution, homogenized at $15,500 \mathrm{rpm}$ for $3 \mathrm{~min}$. The resulting emulsion was centrifuged at $2,000 \mathrm{~g}$ for $5 \mathrm{~min}$ (Hermle Labortechnik, Germany) to eliminate air bubbles. The emulsion was then decanted into glass Petri dishes and left to settle for $24 \mathrm{~h}$ at $37^{\circ} \mathrm{C}$ until the formation of the films.
2.3. Film Thickness. The film thickness was measured with a manual micrometer (Mitutoyo, Japan) with a precision of $0.03 \mathrm{~mm}$. Five measurements were randomly taken for each film. The mean thickness was calculated and used to estimate the WVP and the transparency. The thickness of the films ranged from 0.086 to $0.127 \mathrm{~mm}$.

2.4. Scanning Electron Microscopy (SEM). The surface morphology of the films was examined with a scanning electron microscope (JEOL JSM-5410LV) (Tokyo, Japan) equipped with an INCA system and an EDS (Energy Dispersive XRay) microanalysis detector (Oxford Instruments, Buckinghamshire, UK) and operated at $20 \mathrm{kV}$. For the SEM analysis, the specimens were cut to an adequate size and placed on a cylindrical copper support ( $1 \mathrm{~cm}$ in diameter). The samples were coated with gold to facilitate conduction and prevent the accumulation of charge under electron bombardment. The analysis of the sample was performed under high vacuum conditions.

2.5. Solubility. The solubility determination was performed according to the procedure reported by Casariego et al. [14]. The films were cut into squares $(20 \times 20 \mathrm{~mm})$ and placed in a vacuum oven ( $35 \mathrm{kPa}$ for $24 \mathrm{~h}$ ) to determine the weight of the dry films. Subsequently, the films were immersed in $10 \mathrm{~mL}$ of tridistilled water in Petri dishes kept at $25^{\circ} \mathrm{C}$ for $24 \mathrm{~h}$ with occasional agitation. After this time, the films were dried again in the vacuum oven until they reached constant weight to determine the weight of dry matter that was not dissolved in the water. The solubility was calculated based on

$$
\text { Solubility }=\left(\frac{\mathrm{Mi}-\mathrm{Mf}}{\mathrm{Mi}}\right) 100 \text {, }
$$

where Mi represents the initial mass and Mf the final mass.

2.6. Measurement of the Water Vapor Permeability (WVP). The WVP was determined gravimetrically based on the ASTM E96-92 method [15]. The films were sealed in the upper part of a $50 \mathrm{~mL}$ glass cup, which contained $10 \mathrm{~mL}$ of distilled water $\left(100 \% \mathrm{RH} ; 3.168 \mathrm{kPa}\right.$ of $\mathrm{WVP}$ at $\left.25^{\circ} \mathrm{C}\right)$. Subsequently, they were placed in a desiccator with anhydrous calcium sulfate at $25^{\circ} \mathrm{C}$ and $0 \% \mathrm{RH}(0 \mathrm{kPa}$ of WVP). The cups were weighed at $3 \mathrm{~h}$ intervals for $12 \mathrm{~h}$. The ideal state and uniformity of the WVP conditions were assumed, with a constant air circulation outside of the test cup. For that purpose, a miniature fan was used inside the desiccator. The lost weight of the cup was plotted with respect to time to estimate the slope by a linear equation (the correlation coefficient was 0.99). Once the slope was obtained, the water vapor transmission rate (WVTR) was calculated by

$$
\mathrm{WVTR}=\frac{\text { Slope }}{\text { Film area }} .
$$

The WVP of the films was calculated by multiplying the WVTR by the average thickness of the film $(G)$ and dividing by the difference in partial pressures inside (PA1) and outside (PA2) the cup, as expressed in

$$
\mathrm{WVP}=\frac{\mathrm{WVTR}}{(\mathrm{PA} 1-\mathrm{PA} 2)} G .
$$


2.7. Optical Properties. The color and transparency were determined with a UV-Vis spectrophotometer (Cintra 10E, Australia). For the color measurement, three films of each concentration were chosen, from which the average of five samples was taken. The film color was expressed based on the CIE model $\left(L^{*}, a^{*}\right.$, and $\left.b^{*}\right)$. The transparency was determined according to the method established by Han and Floros [16]. For that purpose, the films were cut into rectangles $(9 \times 45 \mathrm{~mm})$ and placed inside the measuring cell of the spectrophotometer. The transmittance was recorded at $600 \mathrm{~nm}$, and the film transparency was calculated by

$$
\text { Transparency }=\log \left(\frac{T 600}{G}\right),
$$

where $T 600$ is the transmittance at $600 \mathrm{~nm}$ and $G$ represents the film thickness ( $\mathrm{mm}$ ).

The UV-Vis light $(200-800 \mathrm{~nm})$ transmission properties through the films were also determined.

2.8. Antioxidant Activity. The CF-CO (50 mg) was homogenized in $5 \mathrm{~mL}$ of $80 \% \mathrm{v} / \mathrm{v}$ methanol using a homogenizer (Ika Ultra-turrax $\mathrm{T} 18$ basic, Germany) at $5^{\circ} \mathrm{C}$ for $5 \mathrm{~min}$ at $11,000 \mathrm{rpm}$. Afterwards, the sample was centrifuged at $16,000 \mathrm{~g}$ for $10 \mathrm{~min}$ at $5^{\circ} \mathrm{C}$; the supernatant was collected and filtered through Whatman number 1 filter paper. These film extracts were used for the assays of DPPH, ABTS, and the protective effect on human erythrocytes.

The DPPH was determined according to the method described by S. Moein and M. R. Moein [17], with certain modifications. A stock solution was prepared by mixing $2.5 \mathrm{mg}$ of the DPPH radical in $100 \mathrm{~mL}$ of pure methanol and was adjusted to $0.7 \pm 0.02$ at $490 \mathrm{~nm}$ of absorbance. Trolox (6hydroxy-2,5,7,8-tetramethylchroman-2-carboxylic acid) was used as the standard and $80 \%$ methanol as the blank reactant. The sample $(5 \mu \mathrm{L})$ was placed on a microplate, and $245 \mu \mathrm{L}$ of DPPH radical was added. The mixture was kept in the dark for $30 \mathrm{~min}$, and the absorbance was then read in a microplate reader (iMark Bio-Rad, Japan) at a wavelength of $490 \mathrm{~nm}$. The inhibition percentage was calculated for each of the CFCO films, which indicates the capacity of the antioxidant to reduce the radical absorbance after the final incubation. Finally, the results were expressed in trolox equivalents per gram of film (mmol TE/g).

The ABTS test was determined according to Re et al. [18]. The $\mathrm{ABTS}^{+}$radical was generated by the interaction of $5 \mathrm{~mL}$ of ABTS solution $(7 \mathrm{mM})$ and $88 \mu \mathrm{L}$ of potassium persulfate solution $(0.139 \mathrm{mM})$. The test was performed by adding $295 \mu \mathrm{L}$ of the $\mathrm{ABTS}^{+}$solution and $5 \mu \mathrm{L}$ of the film extract. Once the components were mixed in the microplate, the absorbance was monitored at $734 \mathrm{~nm}$. The inhibition percentage was calculated and converted to trolox equivalents (TE) per gram of film ( $\mathrm{mmol} \mathrm{TE} / \mathrm{g})$.

2.9. Evaluation of the Protective Effect on the Human Erythrocyte. The hemolysis induced by AAPH [2,2'-azobis (2amidinopropane hydrochloride)] was determined by the method established by Lu et al. [19] with certain modifications. First, the erythrocytes were washed three times with phosphate-buffered saline (PBS, $\mathrm{pH}=7.4$ ). Subsequently, a 5\% erythrocyte suspension was prepared with PBS. For the erythrocyte protection test, $50 \mu \mathrm{L}$ of erythrocytes at $5 \%$ was placed with $50 \mu \mathrm{L}$ of film extract at $400 \mathrm{mmol}$ in a glass tube. A control mixture was prepared in a similar manner but without the film extract (complete hemolysis). The samples were incubated at $37^{\circ} \mathrm{C}$ with continuous agitation at $30 \mathrm{rpm}$ in the dark for $3 \mathrm{~h}$. After the incubation, the reaction mixture was diluted with $1 \mathrm{~mL}$ of PBS and centrifuged at $2000 \mathrm{~g}$ for $5 \mathrm{~min}$. The supernatant absorbance was read at $540 \mathrm{~nm}$ in a microplate reader (iMark Bio-Rad, Japan). The results were expressed as the hemolysis inhibition percentage in the human erythrocytes, which was calculated using

Hemolysis inhibition percentage

$$
=\left(\frac{\mathrm{AAPH}-\mathrm{HM}}{\mathrm{AAPH}}\right) \times 100
$$

where $\mathrm{AAPH}=$ absorbance with complete hemolysis and HM $=$ absorbance of the supernatant.

2.10. Statistical Analysis. Analysis of variance was applied to the obtained data using CF with and without CO incorporation as factors. The transparency, WVP, solubility, antioxidant, and antihemolytic activity were taken as response variables. For the decision-making process, the Duncan comparison test was used $(\alpha=0.05)$. All of the above analysis was conducted using the NCSS statistical package, 2000.

\section{Results and Discussion}

3.1. Microstructure. The surface appearance of the film microstructure changed upon the addition of cinnamon oil. Figure 1 shows SEM images of the CF alone and with incorporated CO $1.0 \%$, where a surface without pores and with slight folds can be observed in the CF (control). Recently, CF has been reported to lack apparent pores [14]; however, other authors have documented the presence of pores uniformly distributed on the film surface [20]. It is known that, in general, this characteristic depends on the nature of the chitosan (acetylation degree and molecular weight). In contrast, the CFs with $\mathrm{CO}$ presented a rough surface with pores of approximately $2-3 \mu \mathrm{m}$ in diameter. The presence of these pores is likely due to the flocculation and coalescence of small drops of emulsified cinnamon oil during the drying of the film; similar results have been reported by Peng and Li [21] and López-Mata et al. [22] in films with carvacrol incorporated.

3.2. Solubility. The solubility is a very important property of the films that measures their resistance to water. Figure 2 shows the solubility percentages of the CFs with incorporated $\mathrm{CO}$, where it can be observed that the incorporation of CO into the CF significantly decreased $(p<0.05)$ the CF solubility. This decrease was $15 \%$ for CF-CO $0.25 \%$ and $22 \%$ for CF-CO $0.5 \%$ and $1.0 \%$ compared to the control. It was also observed that increasing the $\mathrm{CO}$ concentration from $0.5 \%$ to $1.0 \%$ did not induce greater insolubility of the film. The solubility percentage of the CF (41\%) is consistent with the ranges previously published for CF (16 and 46\%) and reported by Casariego et al. [14]. When working with CF, it is complicated 


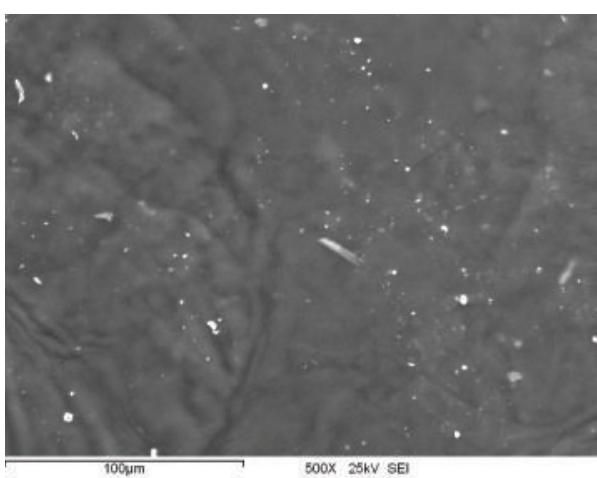

(a)

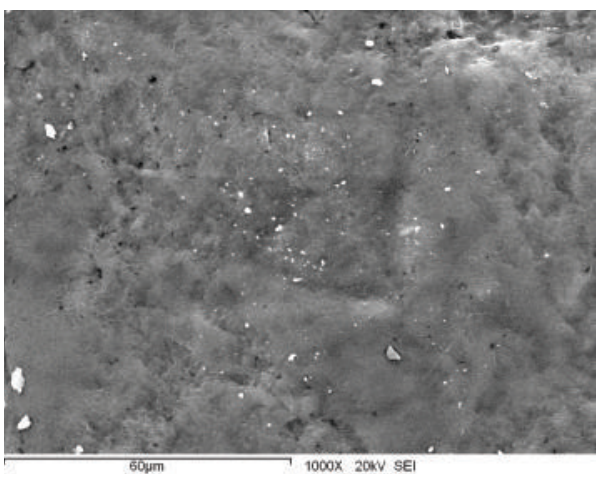

(c)

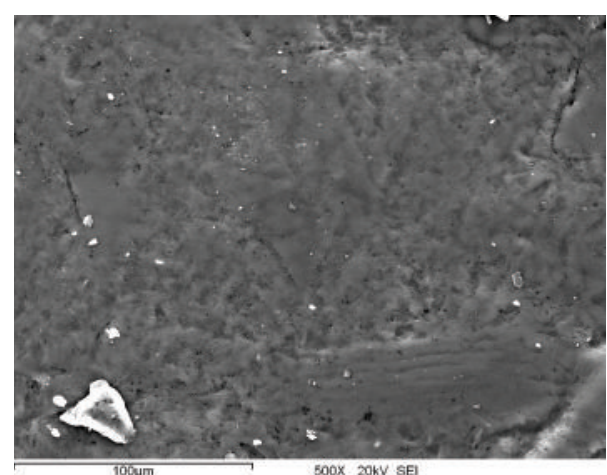

(b)

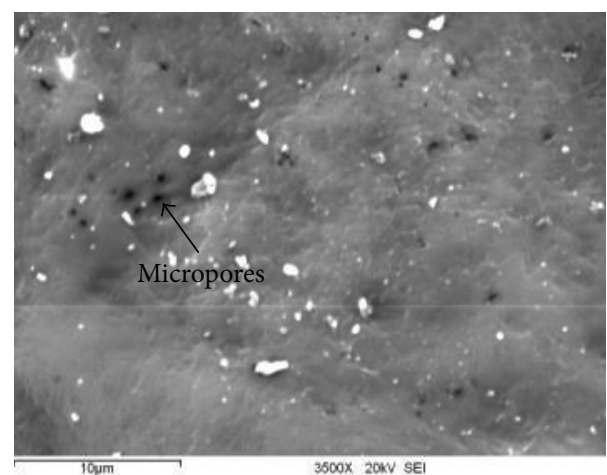

(d)

Figure 1: Scanning Electron Microscopy (SEM) pictures of chitosan film (a) and chitosan film with 1.0\% incorporated cinnamon oil (b, c, and d) oriented on the dry side.

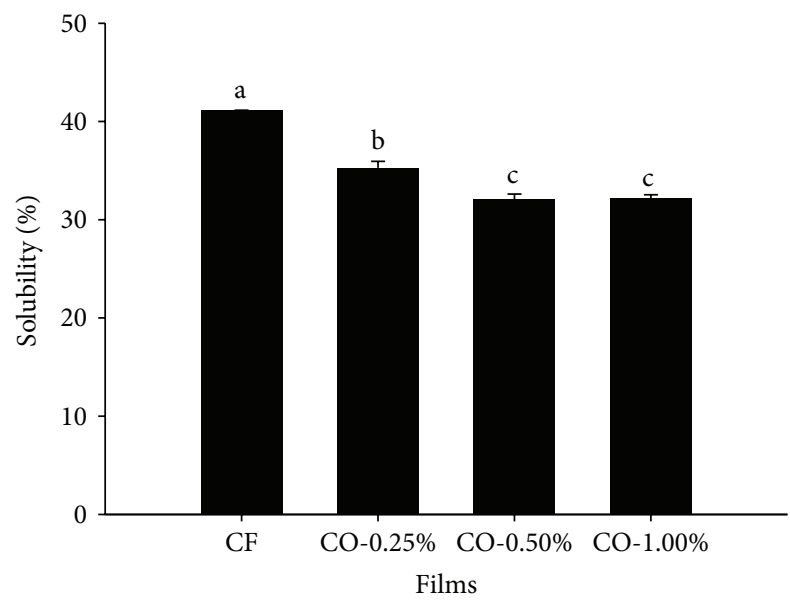

FIGURE 2: Solubility percentages values of CF and CF-CO films.

to standardize a solubility value, as the chitosan properties can vary depending on the source, acetylation degree, and molecular weight. Therefore, the elevated solubility exhibited by the control film could be influenced by the presence of the plasticizer used during the elaboration of the film and the chitosan functional groups [23]. The solubility reduction of the films incorporated with $\mathrm{CO}$ could be due to a decrease in the hydrophobic nature influenced by the loss of its free functional groups (amino and hydroxyl). The low affinity presented by the films with incorporated $\mathrm{CO}$ is important for their application as coatings in food products with high moisture contents.

3.3. Water Vapor Permeability (WVP). The WVP is a property that indicates the rate at which water is lost through a film. The WVP behavior of the studied films is shown in Figure 3. These results show that the incorporation of $0.25 \%$ $\mathrm{CO}$ into CF gives a WVP of $0.53 \pm 0.04 \mathrm{~g} \mathrm{~mm} / \mathrm{kPa} \mathrm{h} \mathrm{m}^{2}$, a $40 \%$ decrease compared to the control $\left(0.88 \mathrm{~g} \mathrm{~mm} / \mathrm{kPa} \mathrm{h} \mathrm{m}^{2}\right)$, which is statistically significant. The incorporation of 0.5 and $1.0 \%$ of $\mathrm{CO}$ into $\mathrm{CF}$ did not show significant difference compared with the control, with values of 0.816 and $1.0178 \mathrm{~g} \mathrm{~mm} / \mathrm{kPa} \mathrm{h} \mathrm{m}^{2}$, respectively. Sánchez-González et al. [1] established that increasing concentrations of essential oils in food films do not necessarily translate to a linear reduction of the WVP. The reduction of the WVP at low concentrations of incorporated $\mathrm{CO}$ could be due to the action of the diverse compounds that compose the $\mathrm{CO}$, which could, as a whole, form a hydrophobic region in the film [24]. However, incrementally increasing the $\mathrm{CO}$ concentration can induce structural changes in the film, as can be observed in Figures 1(a), $1(\mathrm{~b}), 1(\mathrm{c})$, and $1(\mathrm{~d})$, where the control film is compared with the CF-CO $1.0 \%$. Here, a surface with a rougher appearance and with the formation of pores is observed in the case of the CF-CO $1.0 \%$. It has been previously documented that the 


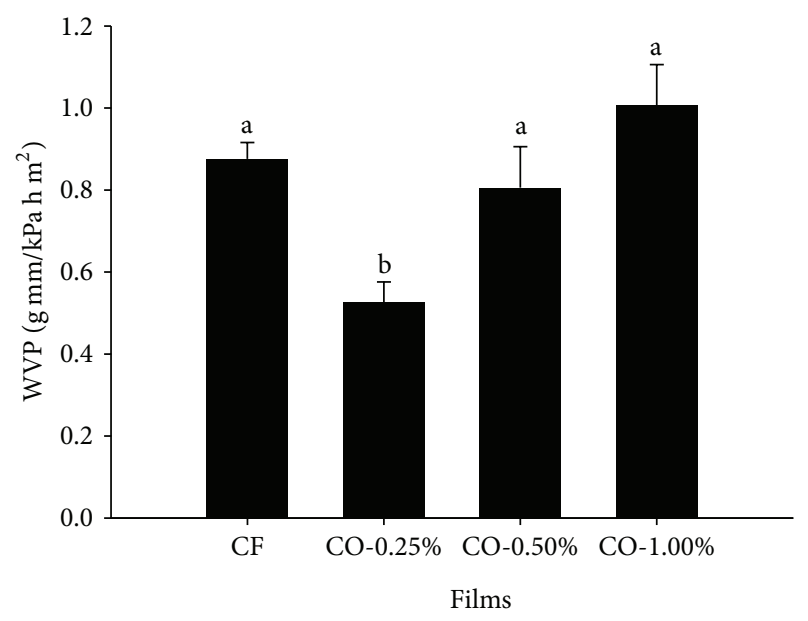

FIgUre 3: Water Vapor Permeability (WVP) of the CF and CF with the incorporation of CO.

introduction of cinnamaldehyde (the main component of the $\mathrm{CO}$ at $\approx 80 \%$ ) into the chitosan structure can react with the amino group of the $\mathrm{C} 2$, inducing the formation of a Schiff base [25]. This base can induce cross-linking of the chitosan polymer, and it has been observed that this cross-linking can induce the presence of pores in the films. Also, several authors have shown the properties of cinnamaldehyde as a cross-linking agent with chitosan [26,27], and these authors reported that the introduction of aldehyde groups in chitosan can induce pore formation in the structure under controlled conditions. The slight increase in WVP of the CF with $1.0 \%$ of $\mathrm{CO}$ could be due to the formation of pores, which could only occur in superficial parts of the film or in a partial manner; all pores do not necessarily form channels that connect both sides of the film. However, it is also possible that these pores arose from the instability of the emulsion drops, which escaped and left an apparent pore trace. These results are similar to the report by Sun et al. [28], who evaluated chitosan film incorporated with various concentrations of complex of $\beta$ cyclodextrin and essential oils as eugenol, cinnamaldehyde, and carvacrol, and Bonilla et al. [29], who evaluated chitosan film with basil essential oil. These authors showed that when the concentration of oils increased, the WVP of the film increased, and this increase was attributed to excessive oils in the films, which subsequently decreased the intermolecular forces between polymer chains that induce segmental motions and free space, causing a more open matrix.

3.4. Color Properties. The measurement of the color parameters is an important property in describing the appearance of films. Table 1 shows the effects of CO incorporation into the CF, where it can be observed that the CF with incorporated $\mathrm{CO}$ generally presented a yellow coloration $\left(b^{*}\right)$. This coloration was significantly lower when $\mathrm{CO}$ was incorporated at concentrations of $0.25 \%$ and $0.5 \%$. However, the yellow coloration in the CF was intensified with a higher concentration of incorporated CO (1.0\%) $(p<0.05)$. Consequently, this phenomenon could be considered normal
TABLE 1: Color parameters of CF and CF-CO films.

\begin{tabular}{lccc}
\hline Films & $L^{*}$ & $a^{*}$ & $b^{*}$ \\
\hline CF & $68.15 \pm 0.73^{\mathrm{a}}$ & $-0.12 \pm 0.04^{\mathrm{a}}$ & $6.25 \pm 0.10^{\mathrm{a}}$ \\
CO-0.25\% & $67.89 \pm 1.00^{\mathrm{a}}$ & $-0.15 \pm 0.02^{\mathrm{a}}$ & $5.44 \pm 0.09^{\mathrm{b}}$ \\
CO-0.50\% & $65.31 \pm 0.99^{\mathrm{a}}$ & $0.14 \pm 0.01^{\mathrm{a}}$ & $5.82 \pm 0.08^{\mathrm{c}}$ \\
CO-1.00\% & $59.53 \pm 0.42^{\mathrm{b}}$ & $0.13 \pm 0.04^{\mathrm{a}}$ & $7.77 \pm 0.05^{\mathrm{d}}$ \\
\hline
\end{tabular}

* The data represent the mean \pm standard deviation of three determinations, and different letters in the same column indicate significant differences $(p<$ $0.05)$.

in the control film, as this coloration has been associated with the presence of repeated units of $\beta$-(1-4)-2-amino-2deoxy-D-glucopyranose [20]. The reduction of the coloration presented in the films with $0.25 \%$ and $0.5 \%$ incorporated $\mathrm{CO}$ could be due to the competition of the diverse $\mathrm{CO}$ compounds when interacting with the functional groups of the CF; however, this effect could also be associated with the presence of the surfactant (Tween 80) used during the elaboration of the film, which can also form uniform structures with the chitosan [30]. Therefore, the increase in the yellow coloration of the CF with $1.0 \%$ CO could be due to the increased cinnamaldehyde concentration and the possible formation of a Schiff base. However, the values of $L^{*}$ (luminosity and brightness) significantly decreased in the CF with $1.0 \%$ CO. This variation in the luminosity induced by the incorporation of $\mathrm{CO}$ could be due to the molecular alteration of chitosan.

The transparency of the film is a desirable property because the consumer needs to clearly see the product covered by the film [31]. The CFs with $0.5 \%$ and $1.0 \%$ $\mathrm{CO}$ incorporated presented greater transparency than the control (Table 2), which indicates that the incorporation of $\mathrm{CO}$ can reduce the opacity of the CF. However, even with this reduction, the values indicate that the films are slightly opaque. Another evaluated parameter was the transmission of light through the film, where it could be observed that the films with incorporated $\mathrm{CO}$ and the control have the property of being able to block ultraviolet radiation in the region of $280 \mathrm{~nm}$, in a transmission \% range of $0.15 \%$ to $0.02 \%$. However, the lowest transmission of the films was in the $200 \mathrm{~nm}$ region $(0.19 \%-0.01 \%)$.

3.5. Antioxidant Activity. The antioxidant activity of the films was measured by two methods, DPPH and ABTS. The combination of two methods to measure the antioxidant activity has been recommended for a better understanding of the extract antioxidant properties. Figure 4 shows the antioxidant activity (DPPH) of the extracts of the studied films. A significant increase in the antioxidant capacity of the CF with CO incorporated at $0.5 \%$ (6.01 times) and $1.0 \%$ (14.5 times) can be observed compared to the control. This activity can be attributed to the presence of the main $\mathrm{CO}$ antioxidant, which is eugenol; however, it could also be due to the presence of cinnamaldehyde, which has been reported to have a lower activity than eugenol, or to a synergetic effect among chitosan, eugenol, and cinnamaldehyde [32]. It has been previously demonstrated that chitosan presents antioxidant activity, which has been attributed to the presence of 
TABLE 2: Light transmittance (\%) and transparency values of CF and CF-CO films.

\begin{tabular}{|c|c|c|c|c|c|c|c|c|c|}
\hline \multirow{2}{*}{ Films } & \multicolumn{8}{|c|}{ Wavelength $(\mathrm{nm})$} & \multirow{2}{*}{ Transparency value* } \\
\hline & 200 & 280 & 350 & 400 & 500 & 600 & 700 & 800 & \\
\hline $\mathrm{CF}$ & 0.19 & 0.15 & 20.36 & 30.46 & 37.25 & 40.20 & 42.09 & 43.47 & $2.66 \pm 0.012^{\mathrm{a}}$ \\
\hline CO- $0.25 \%$ & 0.08 & 0.17 & 16.82 & 28.51 & 34.37 & 36.64 & 38.05 & 39.07 & $2.69 \pm 0.008^{\mathrm{a}}$ \\
\hline CO- $0.50 \%$ & 0.01 & 0.02 & 13.75 & 27.19 & 33.42 & 36.01 & 37.58 & 38.71 & $2.56 \pm 0.010^{\mathrm{d}}$ \\
\hline CO-1.00\% & 0.00 & 0.02 & 5.25 & 18.40 & 25.56 & 28.47 & 30.17 & 31.25 & $2.34 \pm 0.003^{c}$ \\
\hline
\end{tabular}

${ }^{*}$ The data represent the mean \pm standard deviation of three determinations, and different letters in the same column indicate significant differences $(p<0.05)$.

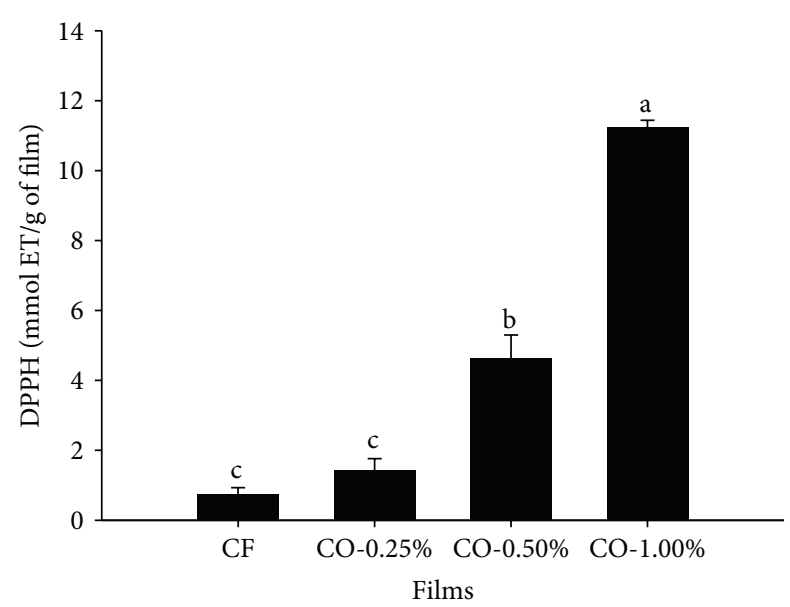

FIGURE 4: Antioxidant capacity of films extract determined by $\mathrm{DPPH}$. The data are the mean values of at least three determinations. The mean values represented by the bars for each type of films extract that are indicated with a different letter are significantly different $(p \leq 0.05)$.

nitrogen located at the $\mathrm{C} 2$ of the polymeric structure [33]. The antioxidant activity tested by ABTS was similar to the results of the DPPH testing method. Therefore, these results show that the antioxidant capacity is increased between 6.1 and 16.4 times for the $\mathrm{CF}$ with $\mathrm{CO}$ incorporated at $0.5 \%$ and $1.0 \%$, respectively, compared to the control (Figure 5).

3.6. Evaluation of the Protective Effect on Human Erythrocytes. For this evaluation, a radical initializer was used (AAPH), which generates peroxyl/alkoxy radicals in the presence of oxygen at physiological temperature. These secondary radicals are responsible for biological damage to cells. Figure 6 shows the protective effects of the CFs with incorporated $\mathrm{CO}$ and the control extracts. In general, all films offered a protective effect to the erythrocytes; this effect provided more than $67 \%$ hemolysis inhibition. The extract of the CFs with $1.0 \%$ CO presented the greatest protective effect, inhibiting hemolysis by up to $80 \%(p<0.05)$. It is important to highlight that the control presented a higher capacity for hemolysis inhibition. Previous studies have shown that chitosan, with its positively charged amino groups, can interact with the negative charges of the erythrocyte membrane and produce a possible chitosan-erythrocyte aggregate that does not seriously damage the erythrocyte membrane [34]. This aggregation most likely forms a net that protect erythrocytes against the

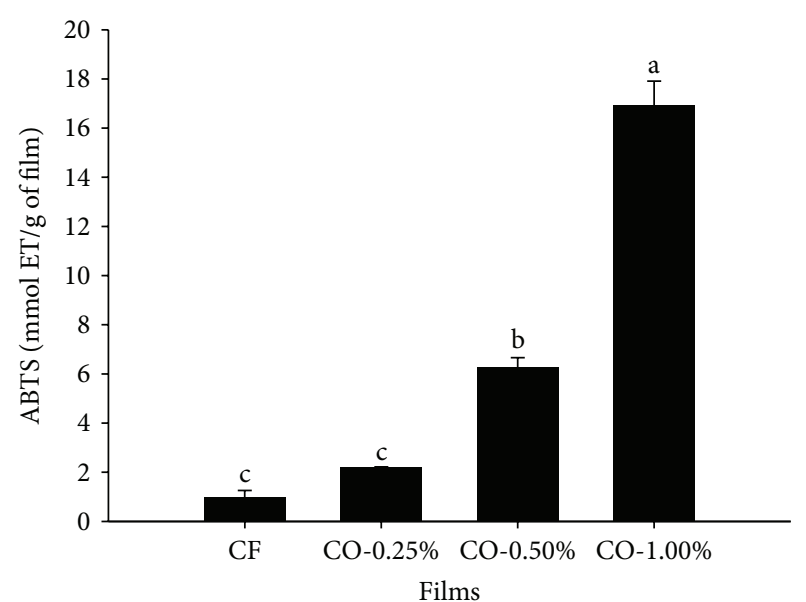

FIGURE 5: Antioxidant capacity of films extract determined by ABTS. The data are the mean values of at least three determinations. The mean values represented by the bars for each type of films extract that are indicated with a different letter are significantly different $(p \leq 0.05)$.

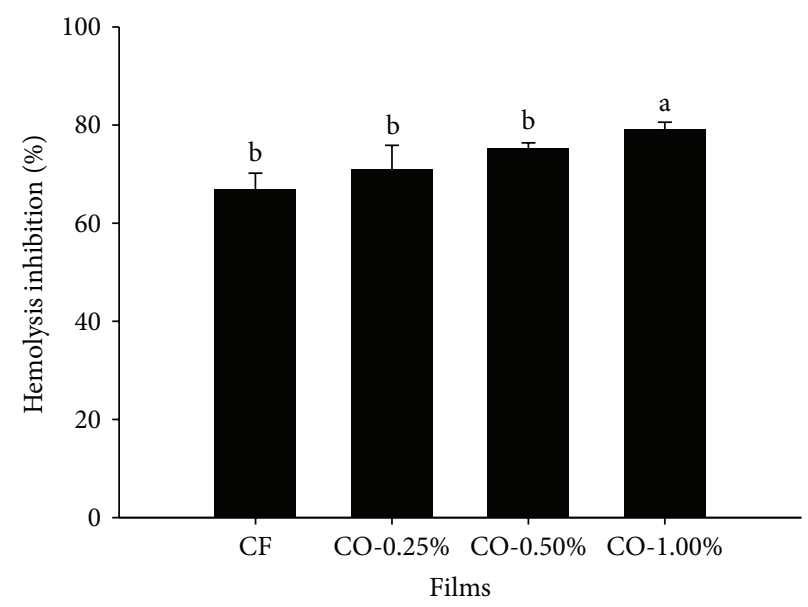

FIGURE 6: Antioxidant capacity of films extract determined by percentage of hemolysis inhibition. The data are the mean values of at least three determinations. The mean values represented by the bars for each type of films extract that are indicated with a different letter are significantly different $(p \leq 0.05)$.

attacks of radicals generated by the AAPH. In the case of the CF extract with the highest incorporated CO concentration, its greater hemolysis inhibition effect could be associated 
with the probable presence of other biomolecules that might inhibit the radicals, in addition to the possible protective net formed by the chitosan-erythrocyte charges.

\section{Conclusions}

In this study, multiple concentrations of $\mathrm{CO}$ were incorporated into CFs, and their physical and antioxidant properties were evaluated. The incorporation of $\mathrm{CO}$ was observed to decrease the CF solubility, while WVP was only reduced at low incorporated CO concentrations (0.25\%). It was also observed that all films presented a yellow coloration, which was reduced at low incorporated $\mathrm{CO}$ concentrations but accentuated at incorporated CO concentrations of $1.0 \%$. Regarding the transparency, it was observed that the incorporation of $\mathrm{CO}$ can improve the transparency of the films and also act as a good blocker of ultraviolet radiation. The antioxidant activity of the CF incorporated with CO strengthened the antioxidant activity of the films by 6.0 to 14.5 times compared to the control, and the films with $1.0 \%$ CO incorporated could protect erythrocytes against free radical attack by up to $80 \%$.

\section{Conflict of Interests}

The authors declare that there is no conflict of interests regarding the publication of this paper.

\section{Acknowledgments}

Financial support from PROMEP (ITSON-PTC-056 and 103.5/12/2051) and ITSON (PROFAPI 0270 and 00401) is gratefully acknowledged.

\section{References}

[1] L. Sánchez-González, M. Vargas, C. González-Martínez, A. Chiralt, and M. Cháfer, "Use of essential oils in bioactive edible coatings: a review," Food Engineering Reviews, vol. 3, no. 1, pp. $1-16,2011$

[2] D. Lin and Y. Zhao, "Innovations in the development and application of edible coatings for fresh and minimally processed fruits and vegetables," Comprehensive Reviews in Food Science and Food Safety, vol. 6, no. 3, pp. 60-75, 2007.

[3] H. K. No, S. P. Meyers, W. Prinyawiwatkul, and Z. Xu, "Applications of chitosan for improvement of quality and shelf life of foods: a review," Journal of Food Science, vol. 72, no. 5, pp. R87R100, 2007.

[4] G. Cárdenas, P. Anaya, C. von Plessing, C. Rojas, and J. Sepúlveda, "Chitosan composite films. Biomedical applications," Journal of Materials Science: Materials in Medicine, vol. 19, no. 6, pp. 2397-2405, 2008.

[5] G. A. González-Aguilar, E. Valenzuela-Soto, J. Lizardi-Mendoza et al., "Effect of chitosan coating in preventing deterioration and preserving the quality of fresh-cut papaya 'Maradol', Journal of the Science of Food and Agriculture, vol. 89, no. 1, pp. 15-23, 2009.

[6] M. Friedman and V. K. Juneja, "Review of antimicrobial and antioxidative activities of chitosans in food," Journal of Food Protection, vol. 73, no. 9, pp. 1737-1761, 2010.
[7] C. A. Campos, L. N. Gerschenson, and S. K. Flores, "Development of edible films and coatings with antimicrobial activity," Food and Bioprocess Technology, vol. 4, no. 6, pp. 849-875, 2011.

[8] V. Giatrakou, A. Ntzimani, and I. N. Savvaidis, "Combined chitosan-thyme treatments with modified atmosphere packaging on a ready-to-cook poultry product," Journal of Food Protection, vol. 73, no. 4, pp. 663-669, 2010.

[9] Y. Ding, E. Q. Wu, C. Liang et al., "Discrimination of cinnamon bark and cinnamon twig samples sourced from various countries using HPLC-based fingerprint analysis," Food Chemistry, vol. 127, no. 2, pp. 755-760, 2011.

[10] L. Shojun, C. Freitag, and J. J. Morrell, "Preventing fungal attack of freshly sawn lumber using cinnamon extracts," Forest Products Journal, vol. 58, no. 7-8, pp. 77-81, 2008.

[11] B. Shan, Y.-Z. Cai, J. D. Brooks, and H. Corke, "Antibacterial properties and major bioactive components of cinnamon stick (Cinnamomum burmannii): activity against foodborne pathogenic bacteria," Journal of Agricultural and Food Chemistry, vol. 55, no. 14, pp. 5484-5490, 2007.

[12] C. L. del Toro-Sánchez, J. F. Ayala-Zavala, L. Machi et al., "Controlled release of antifungal volatiles of thyme essential oil from $\beta$-cyclodextrin capsules," Journal of Inclusion Phenomena and Macrocyclic Chemistry, vol. 67, no. 3, pp. 431-441, 2010.

[13] T. Hongpattarakere and O. Riyaphan, "Effect of deacetylation conditions on antimicrobial activity of chitosans prepared from carapace of black tiger shrimp (Penaeus monodon)," Songklanakarin Journal of Science and Technology, vol. 30, no. 1, pp. $1-9,2008$.

[14] A. Casariego, B. W. S. Souza, M. A. Cerqueira et al., "Chitosan/ clay films' properties as affected by biopolymer and clay micro/ nanoparticles' concentrations," Food Hydrocolloids, vol. 23, no. 7, pp. 1895-1902, 2009.

[15] V. Guillard, B. Broyart, C. Bonazzi, S. Guilbert, and N. Gontard, "Preventing moisture transfer in a composite food using edible films: experimental and mathematical study," Journal of Food Science, vol. 68, no. 7, pp. 2267-2277, 2003.

[16] J. H. Han and J. D. Floros, "Casting antimicrobial packaging films and measuring their physical properties and antimicrobial activity," Journal of Plastic Film and Sheeting, vol. 13, no. 4, pp. 287-298, 1997.

[17] S. Moein and M. R. Moein, "Relationship between antioxidant properties and phenolics in Zhumeria majdae," Journal of Medicinal Plants Research, vol. 4, no. 7, pp. 517-521, 2010.

[18] R. Re, N. Pellegrini, A. Proteggente, A. Pannala, M. Yang, and C. Rice-Evans, "Antioxidant activity applying an improved ABTS radical cation decolorization assay," Free Radical Biology and Medicine, vol. 26, no. 9-10, pp. 1231-1237, 1999.

[19] J. Lu, Y. Jin, G. Liu et al., "Flavonoids from the leaves of actinidia kolomikta," Chemistry of Natural Compounds, vol. 46, no. 2, pp. 205-208, 2010.

[20] M. Pereda, A. G. Ponce, N. E. Marcovich, R. A. Ruseckaite, and J. F. Martucci, "Chitosan-gelatin composites and bi-layer films with potential antimicrobial activity," Food Hydrocolloids, vol. 25, no. 5, pp. 1372-1381, 2011.

[21] Y. Peng and Y. Li, "Combined effects of two kinds of essential oils on physical, mechanical and structural properties of chitosan films," Food Hydrocolloids, vol. 36, pp. 287-293, 2014.

[22] M. A. López-Mata, S. Ruiz-Cruz, N. P. Silva-Beltrán, J. D. J. Ornelas-Paz, P. B. Zamudio-Flores, and S. E. Burruel-Ibarra, "Physicochemical, antimicrobial and antioxidant properties of chitosan films incorporated with carvacrol," Molecules, vol. 18, no. 11, pp. 13735-13753, 2013. 
[23] S. Y. Park, K. S. Marsh, and J. W. Rhim, "Characteristics of different molecular weight chitosan films affected by the type of organic solvents," Journal of Food Science, vol. 67, no. 1, pp. 194197, 2002.

[24] I. Leceta, P. Guerrero, and K. de la Caba, "Functional properties of chitosan-based films," Carbohydrate Polymers, vol. 93, no. 1, pp. 339-346, 2013.

[25] E. P. Azevedo and V. Kumar, "Rheological, water uptake and controlled release properties of a novel self-gelling aldehyde functionalized chitosan," Carbohydrate Polymers, vol. 90, no. 2, pp. 894-900, 2012.

[26] S. M. Ojagh, M. Rezaei, S. H. Razavi, and S. M. H. Hosseini, "Development and evaluation of a novel biodegradable film made from chitosan and cinnamon essential oil with low affinity toward water," Food Chemistry, vol. 122, no. 1, pp. 161-166, 2010.

[27] L. Higueras, G. López-Carballo, R. Gavara, and P. HernándezMuñoz, "Reversible covalent immobilization of cinnamaldehyde on chitosan films via schiff base formation and their application in active food packaging," Food and Bioprocess Technology, vol. 8, no. 3, pp. 526-538, 2015.

[28] X. Sun, S. Sui, C. Ference et al., "Antimicrobial and mechanical properties of $\beta$-cyclodextrin inclusion with essential oils in chitosan films," Journal of Agricultural and Food Chemistry, vol. 62, no. 35, pp. 8914-8918, 2014.

[29] J. Bonilla, M. Vargas, L. Atarés, and A. Chiralt, "Physical properties of chitosan-basil essential oil edible films as affected by oil content and homogenization conditions," Procedia Food Science, vol. 1, pp. 50-56, 2011.

[30] Y. Peng, L. Yin, and Y. Li, “Combined effects of lemon essential oil and surfactants on physical and structural properties of chitosan films," International Journal of Food Science \& Technology, vol. 48, no. 1, pp. 44-50, 2013.

[31] C. T. Jutaporn, C. Suphitchaya, and W. Thawien, "Antimicrobial activity and characteristics of edible films incorporated with Phayom wood (Shorea tolura) extract," International Food Research Journal, vol. 18, no. 1, pp. 39-54, 2011.

[32] S. Mathew and T. E. Abraham, "Studies on the antioxidant activities of cinnamon (Cinnamomum verum) bark extracts, through various in vitro models," Food Chemistry, vol. 94, no. 4, pp. 520-528, 2006.

[33] P. J. Park, J. Y. Je, and S. K. Kim, "Free radical scavenging activities of differently deacetylated chitosans using an ESR spectrometer," Carbohydrate Polymers, vol. 55, no. 1, pp. 17-22, 2004.

[34] S. Hirano, M. Zhang, M. Nakagawa, and T. Miyata, "Wet spun chitosan-collagen fibers, their chemical N-modifications, and blood compatibility," Biomaterials, vol. 21, no. 10, pp. 997-1003, 2000 . 

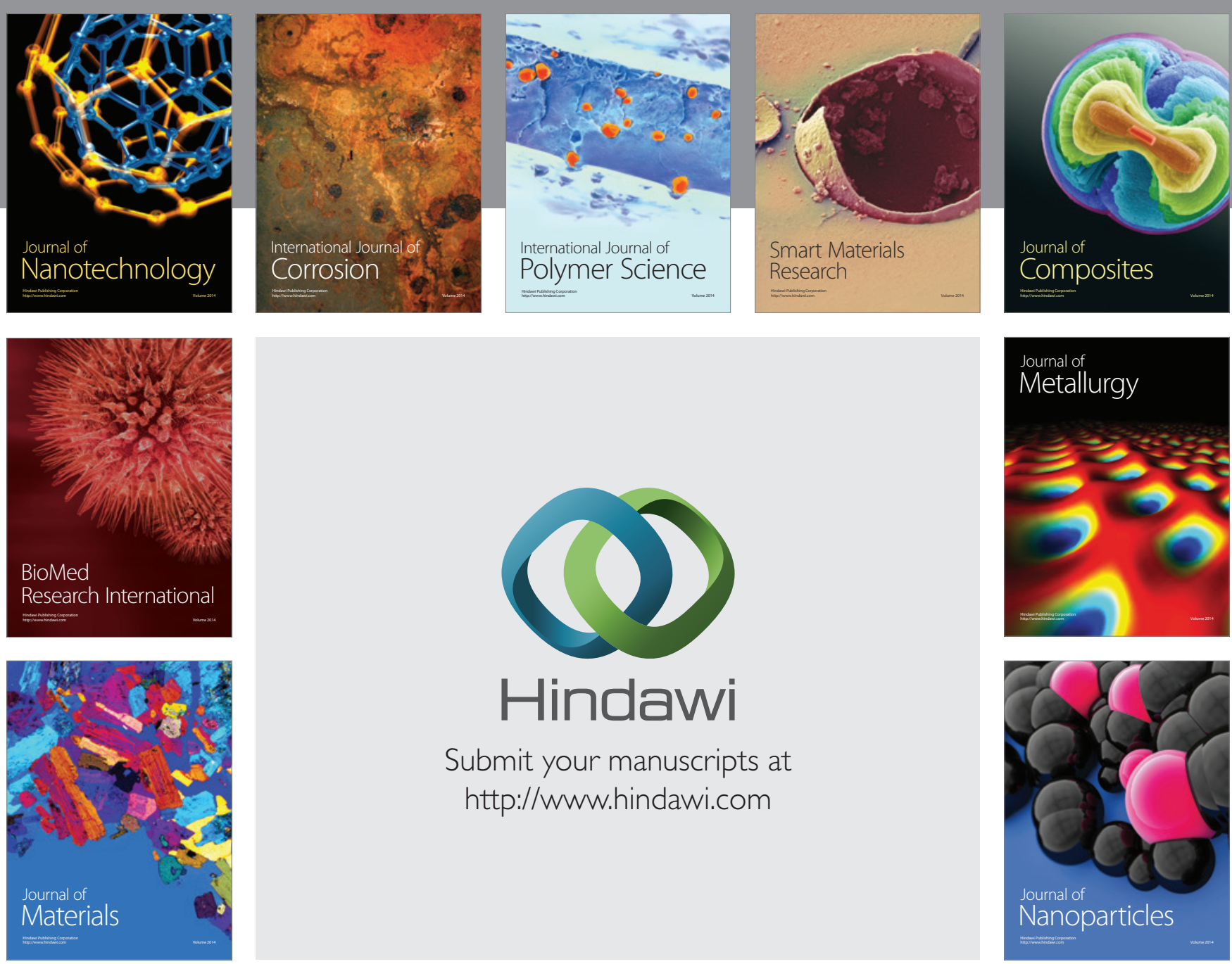

Submit your manuscripts at http://www.hindawi.com
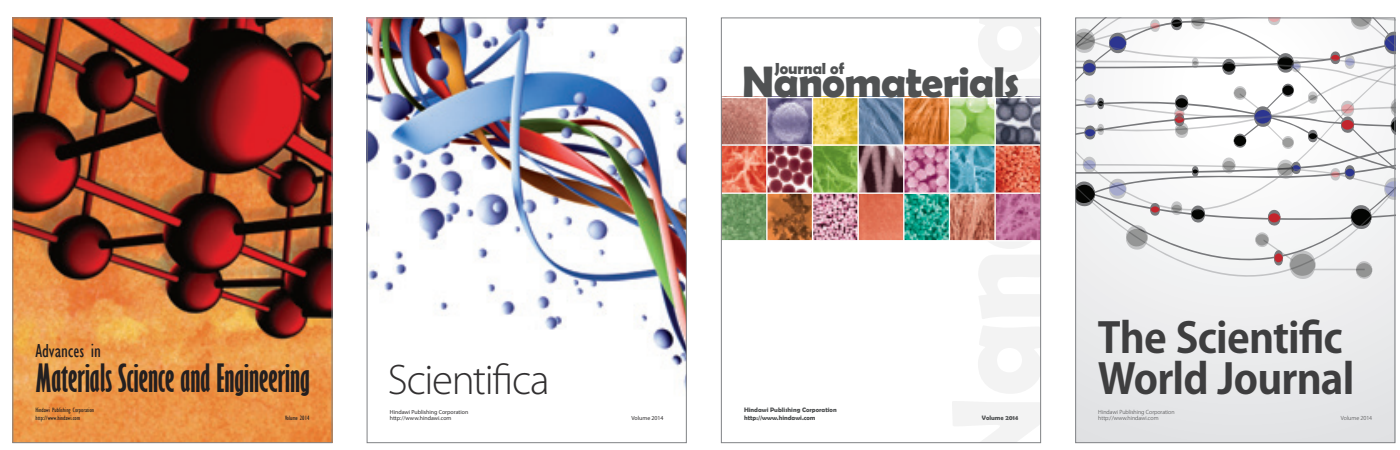

\section{The Scientific World Journal}
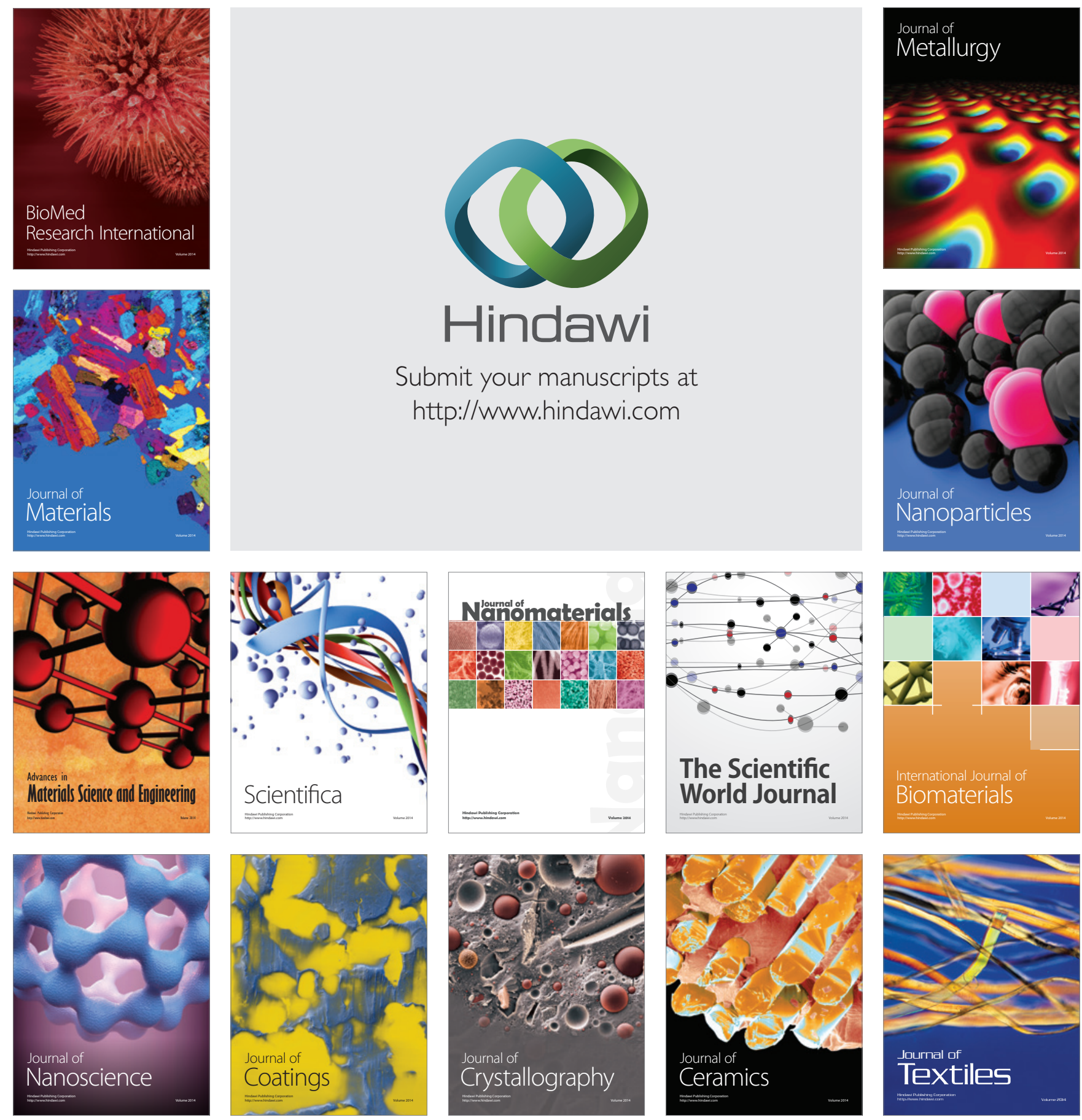